\title{
Degrees of Freedom in Wireless Multiuser Spatial Multiplex Systems With Multiple Antennas
}

\author{
Wei Yu, Member, IEEE, and Wonjong Rhee, Member, IEEE
}

\begin{abstract}
This letter investigates the structure of the optimal spatial multiplex scheme in a multiuser multiantenna wireless fading environment. Based on a sum-capacity criterion, this letter shows that the optimal transmission strategy in an uplink or downlink channel with $n$ antennas at the base-station involves more than $n$ users at the same time. In particular, when remote users are equipped with $m$ antennas each, the maximum number of data streams is shown to be upper bounded by $n^{2}$, with each user transmitting or receiving up to $\mathrm{m}^{2}$ data streams. This gives a dimension-counting interpretation for multiuser diversity. Multiple antennas at the base-station increases the total number of dimensions, thus allowing more users to transmit and receive at the same time. By contrast, multiple antennas at the remote terminal allow a single user to occupy multiple dimensions, which increases its transmission rate, but also has the potential effect of precluding simultaneous transmission by other users.
\end{abstract}

Index Terms-Broadcast channel, multiple-access channel, multiple-input multiple-output (MIMO) system, multiuser diversity, spatial diversity, spatial multiplex.

\section{INTRODUCTION}

$\mathbf{T}$ $\mathrm{HE}$ emergence of multiuser transmission techniques for wireless multiantenna systems has opened up the possibility of allowing multiple users to access the base-station at the same frequency and at the same time. The design of such a multiuser multiple-input multiple-output (MIMO) system demands not only multiuser detection and multiuser precoding techniques at the signal-processing level, but also the implementation of multiuser scheduling algorithms at the system level. The focus of this letter is on the optimal structure of multiuser scheduling and power control in a multiantenna wireless system.

Multiple antennas enhance the performance of wireless systems by creating multiple dimensions in the spatial domain. Spatial dimensions can be used to provide diversity and/or opportunities for spatial multiplex. Consider a cellular system with one base-station and many remote terminals geographically scattered in a single cell. Diversity and spatial multiplex can be understood as follows: As not all remote terminals are likely to experience deep fades at the same time, the total throughput of the multiuser system can be made resilient to channel fading by intelligently selecting a best subset of users. Thus, diversity in

Manuscript received April 19, 2004; revised June 15, 2005 and January 15, 2006. This work was supported in part by the Natural Science and Engineering Research Council (NSERC) of Canada and in part by the Canada Research Chairs program. This paper was presented in part at the IEEE International Conference on Communications (ICC), Helsinki, Finland, 2001, and in part at the IEEE Global Telecommunications Conference (Globecom), San Francisco, CA, 2003.

W. Yu is with the Edward S. Rogers Sr. Department of Electrical and Computer Engineering, University of Toronto, Toronto, ON M5S 3G4, Canada (e-mail: weiyu@ @omm.utoronto.ca).

W. Rhee is with ASSIA Inc., Redwood Shores Pkwy, CA 94065 USA (e-mail: wonjong@dsl.stanford.edu).

Digital Object Identifier 10.1109/TCOMM.2006.881357 a multiuser system occurs not only across the antennas within each user, but also across the set of all users. This notion is often referred to as multiuser diversity. Multiuser diversity was first introduced by Knopp and Humblet [1] and has since been explored by various authors (e.g., [2]-[4]). Note that multiuser diversity differs from single-user spatial diversity in one aspect. In a single-user system, spatial diversity refers to the ability for the multiple antennas to transmit or receive the same information across several paths, while in a multiuser system, independent information is transmitted and received by different users. Thus, a system that uses multiuser diversity may also operate in a spatial multiplex mode whenever more than one user are active at the same time.

The notion of multiuser diversity opens up the following set of questions. In a spatial multiplex system with multiple antennas at the base-station, how many users should be active at any given time? How should the optimal set of users be chosen? What are the appropriate power level and data rate for each user? The answers to these questions would provide useful insights to the design of both the physical layer and the mediumaccess-control layer in a wireless network. This letter tackles these questions by investigating the structure of the sum-capacity-achieving power-allocation strategy in uplink and downlink channels. The optimal power-allocation strategy not only gives a solution to the optimal multiuser scheduling problem, but also sheds light on the interaction between spatial diversity and multiuser diversity.

\section{Multiuser Multiantenna Fading Channel}

\section{A. Uplink Channel Model}

Consider a cellular wireless environment with a base-station equipped with $n$ antennas and $K$ remote users, each equipped with $m$ antennas. The remote-to-base-station transmission can be modeled as follows:

$$
\mathbf{y}(i)=\sum_{k=1}^{K} H_{k}(i) \mathbf{x}_{k}(i)+\mathbf{n}(i)
$$

where $k$ is the user index, and $i$ is the time index. The input signals $\mathbf{x}_{\mathbf{k}}(i)$ are $m$-dimensional vectors; the received signal $\mathbf{y}(i)$ is an $n$-dimensional vector; $\mathbf{n}(i)$ is the independent, identically distributed (i.i.d.) complex Gaussian noise. The user $k$ 's channel at time instant $i$ is represented by $H_{k}(i)$, which is an $n \times m$ matrix with complex entries. This letter assumes an i.i.d. fading model with instantaneous channel state information (CSI) available to all the transmitters and the receiver. The availability of CSI is crucial for power control; in practice, it must be estimated at the receiver and transmitted back to the transmitter by a reliable feedback mechanism. For simplicity, the entries of $H_{k}$ are 
assumed to be i.i.d. complex Gaussian random variables. This corresponds to a Rayleigh channel model with rich scatterers.

The capacity of a $K$-user multiple-access channel is a $K$-dimensional convex region whose boundary points characterize the tradeoff among data rates for various users. The focus of this letter is the maximum $K$-user sum rate. Although it ignores the issue of fairness, the sum-capacity measure is an effective overall figure of merit for the system. It has an efficient numerical characterization [5] and has been the subject of many previous studies.

Ergodic capacity is the channel capacity in the traditional Shannon sense. In this case, channel coding is done over a block length sufficiently large to cover all fading states. The capacity for the multiple-access channel is

$$
C^{(u)}=\max \mathbf{E}_{H_{1}, \ldots, H_{K}} I\left(X_{1}, \ldots, X_{K} ; Y\right)
$$

where the expectation is over the joint channel distribution. The mutual information here is a random variable, which depends on the channel in two ways. First, the explicit computation of $I\left(X_{1}, \ldots, X_{K} ; Y\right)$ depends on the channel. Second, because of the perfect transmitter side-information assumption, the optimal input distribution for $\left(X_{1}, \ldots, X_{K}\right)$ is also a function of $\left(H_{1}, \ldots, H_{K}\right)$. The maximization is over all such input distributions, which are called power-allocation policies.

Gaussian signaling is optimal in the i.i.d. fading multiple-access channel. Let $S_{k}\left(H_{1}, \ldots, H_{K}\right)$ be the $m \times m$ signal covariance matrix for user $k$ at the given channel realization. An uplink power-allocation policy for user $k$ is a mapping

$$
\mathcal{P}_{k}:\left(H_{1}, \ldots, H_{K}\right) \mapsto S_{k}\left(H_{1}, \ldots, H_{K}\right)
$$

The average power constraint $P_{k}$ for user $k$ is satisfied when

$$
\mathbf{E}_{H_{1}, \ldots, H_{K}}\left[\operatorname{tr}\left(S_{k}\left(H_{1}, \ldots, H_{K}\right)\right)\right] \leq P_{k}
$$

where "tr" denotes matrix trace. The optimal sum-capacity point is the solution to the following optimization problem:

$$
\max _{S_{1}, \ldots, S_{K}} \mathbf{E}_{H_{1}, \ldots, H_{K}} I\left(X_{1}, \ldots, X_{K} ; Y \mid H_{1}, \ldots, H_{K}\right)
$$

subject to the average power (or trace) constraints $\left(P_{1}, \ldots, P_{K}\right)$ on $\left(S_{1}, \ldots, S_{K}\right)$.

\section{B. Downlink Channel Model}

The power-control problem for downlink transmission can likewise be defined as follows. The base-station-to-remote-terminal channel is modeled as

$$
\mathbf{y}_{k}^{\prime}(i)=H_{k}^{\prime}(i) \mathbf{x}^{\prime}(i)+\mathbf{n}_{k}^{\prime}(i), \quad k=1, \ldots K
$$

where $\mathbf{x}^{\prime}(i)$ is an $n$-dimensional vector representing the transmitted signal from the base-station, $\mathbf{y}_{k}^{\prime}(i)$ is an $m$-dimensional vector representing the received signal for the $k$ th user, and $\mathbf{n}_{k}^{\prime}(i)$ is the i.i.d. complex Gaussian noise. Again, $H_{k}^{\prime}(i)$ is an $m \times n$ matrix with complex entries, which denotes the channel for user $k$ at time instant $i$. Note that the base-station jointly encodes independent information for all users, while remote terminals are scattered geographically and do not cooperate.
Given the instantaneous channel realization $\left(H_{1}, \ldots, H_{K}\right)$, a downlink power-allocation policy is a mapping

$$
\mathcal{P}^{\prime}:\left(H_{1}, \ldots, H_{K}\right) \mapsto S^{\prime}\left(H_{1}, \ldots, H_{K}\right)
$$

where $S^{\prime}\left(H_{1}, \ldots, H_{K}\right)$ is the transmit covariance matrix. The sum-rate optimum downlink transmission strategy is a powercontrol policy that maximizes the sum capacity of the broadcast channel subject to an average power constraint $P^{\prime}$

$$
\mathbf{E}_{H_{1}, \ldots, H_{K}}\left[\operatorname{tr}\left(S^{\prime}\left(H_{1}, \ldots, H_{K}\right)\right)\right] \leq P^{\prime}
$$

The sum capacity of the broadcast channel is not a simple mutual-information expression. However, it turns out that via a so-called uplink-downlink duality, the broadcast channel problem can be transformed into a multiple-access channel problem. Further, both uplink and downlink problems have the same solution.

\section{Main Contribution}

Intuitively, as each user experiences a different channelfading state, the sum-rate maximizing strategy should involve only a subset of good channels in each time instant. This is true not only in the downlink, where the base-station is free to allocate power to the best users in each time instant, but also in the uplink direction, because each user has the freedom to choose the best time instants to transmit. The objective of this letter is to give a rigorous analysis of the structure of the optimal power-control algorithm and to provide a theoretical upper bound on the maximum number of active users. The main result of this letter is that in a multiuser multiantenna fading channel with $n$ antennas at the base-station, the sum-capacity-achieving transmission scheme in both uplink and downlink directions involves more than $n$ data streams at the same time. In particular, when remote users are equipped with $m$ receive antennas each, the optimal transmission scheme may involve up to $n^{2}$ data streams in total, with each remote terminal using up to $\mathrm{m}^{2}$ data streams.

\section{OPTIMAL UPLINK POWER CONTROL}

\section{A. Simultaneous Waterfilling}

We start the development by considering first the uplink direction. Let $\nu$ be a random variable representing the channelfading state with a cumulative density function $\rho(\nu)$. Denote the channel-fading distribution as $H_{k}(\nu)$, and the power-allocation strategy as $S_{k}(\nu)$. The ergodic sum-capacity maximization problem (5) can be written down as the following convex optimization problem:

$$
\begin{array}{ll}
\max _{S_{k}(\nu)} & \int_{\nu} \log \left|\sum_{k=1}^{K} H_{k}(\nu) S_{k}(\nu) H_{k}^{*}(\nu)+I\right| d \rho(\nu) \\
\text { s.t } \quad & \int_{\nu} \operatorname{tr}\left(S_{k}(\nu)\right) d \rho(\nu) \leq P_{k} \\
& S_{k}(\nu) \geq 0
\end{array}
$$


where $|\cdot|$ denotes matrix determinant, $(\cdot)^{*}$ denote matrix conjugate transpose, " $\geq$ " denotes a matrix positive-semidefiniteness constraint, the noise covariance matrix is assumed to be an identity matrix. Equations (10) and (11) need to be satisfied for all $k=1, \ldots, K$ and for all fading states $\nu$.

In a single-user channel (i.e., $K=1$ ), it is well known that the optimal transmit-covariance matrix $S(\nu)$ is the waterfilling covariance over the set of singular values of the channel $H(\nu)$, both in space and in time. A generalization of this idea to the multiuser setting is that the optimal solution $S_{k}(\nu)$ must satisfy the waterfilling condition over both space and time for every user simultaneously.

Consider the optimization problem (9). Clearly, the global optimal solution $\left\{S_{k}(\nu)\right\}_{k=1}^{K}$ must be such that each $S_{k}(\nu)$ is a single-user "waterfilling" power allocation against the noise and the combined interference from all other users, as otherwise, $S_{k}(\nu)$ can be optimized to improve the global objective. This must be true for $S_{k}(\nu)$ for each $k=1, \ldots, K$. Conversely, if the single-user waterfilling condition is satisfied for every user $k=1, \ldots, K$, because the optimization problem is concave over the positive-semidefinite matrices $S_{k}(\nu)$, the local optimum is also guaranteed to be globally optimal. Thus, the set of $S_{k}(\nu)$ is the solution to (10) if and only if each $S_{k}(\nu)$ is the single-user waterfilling covariance against combined interference and noise.

The above discussion can be made rigorous by examining the Karush-Kuhn-Tucker (KKT) condition for the optimization problem. Associate dual variables $\lambda_{k}$ with each power constraint and $\Phi_{k}(\nu)$ with each positivity constraint, where $\lambda_{k}$ is a scalar, and $\Phi_{k}(\nu)$ are $n \times n$ matrices. It is clear that the following set of KKT conditions need to be satisfied for each fading state $\nu$ and for each user $k$ :

$$
\begin{aligned}
& H_{k}^{*}(\nu)\left(\sum_{j=1}^{K} H_{j}(\nu) S_{j}(\nu) H_{j}^{*}(\nu)+I\right)^{-1} H_{k}(\nu)+\Phi_{k}(\nu)=\lambda_{k} I \\
& \quad \int_{\nu} \operatorname{tr}\left(S_{k}(\nu)\right) d \rho(\nu) \leq P_{k} \\
& \operatorname{tr}\left(\Phi_{k}(\nu) S_{k}(\nu)\right)=0 \\
& \quad \Phi_{k}(\nu), S_{k}(\nu), \lambda_{k} \geq 0
\end{aligned}
$$

This set of KKT conditions is a straightforward generalization of a similar result in [5], where the nonfading case is treated. Note that the power-control strategy is a function of the channel fading state, but the waterfilling level $\lambda_{k}$ is a function of the fading distribution only, which can be precomputed.

In fact, as shown in [5], this simultaneous waterfilling condition leads to an iterative waterfilling procedure to compute the optimal $S_{k}(\nu)$. Starting from any initial power allocation, each step of the iterative procedure consists of a single-user waterfilling for $S_{k}(\nu)$ with all other users' signals regarded as noise. The set of covariance matrices $S_{k}(\nu)$ eventually converges to the optimum solution to (10).

\section{B. Single-Antenna Case}

When there is only one antenna for each transmitter and for the receiver, [1] showed that the sum-rate maximizing power- control strategy is a time-division multiple-access (TDMA)-like strategy where a single user with the best channel transmits at every moment. This result can be rederived using the simultaneous waterfilling interpretation. This sets the stage for subsequent development where multiple antennas are introduced.

In the single-antenna case, the KKT condition simplifies considerably. In particular, (12) reduces to a scalar equation with scalar variables $s_{j}(\nu)$ and $\phi_{k}(\nu)$. Without the $\phi_{k}(\nu)$ term, (12) is the familiar waterfilling condition with water level equal to $\lambda_{k}^{-1}$. The variable $\phi_{k}(\nu)$ is used to account for the possibility that a fading state may be sufficiently weak so that no power is allocated for that state. In that case, a positive $\phi_{k}(\nu)$ is used to make up the difference. The slack variable can only be nonzero when $s_{k}(\nu)=0$.

Now, if two users $k$ and $l$ both transmit at a fading state $\nu$, they must both satisfy the single-user waterfilling condition

$$
\begin{aligned}
\lambda_{k} & =\frac{h_{k}^{2}(\nu)}{\sum_{j=1}^{K} h_{j}^{2}(\nu) s_{j}(\nu)+\sigma^{2}} \\
\lambda_{l} & =\frac{h_{l}^{2}(\nu)}{\sum_{j=1}^{K} h_{j}^{2}(\nu) s_{j}(\nu)+\sigma^{2}} .
\end{aligned}
$$

The denominator for the two conditions are the same, so if both users transmit, then

$$
\frac{h_{k}^{2}(\nu)}{\lambda_{k}}=\frac{h_{l}^{2}(\nu)}{\lambda_{l}} .
$$

In other words, the fading state $\nu$ may not be shared by the two users unless the channel gains differ by exactly the factor $\lambda_{k} / \lambda_{l}$. Since the channel fading state is assumed to be i.i.d. complex Gaussian distributed, such an event has zero probability. Therefore, we have proved the following.

Lemma 1 (Knopp and Humblet [1]): In a single-antenna multiple-access fading channel with i.i.d. Gaussian fading statistics, assuming perfect side information at the transmitters and the receiver, with probability one, the sum capacity is achieved with a power-control strategy that allows only one user to transmit at a time.

This same conclusion was reached earlier by Cheng and Verdu [6] in the context of a multiple-access channel with intersymbol interference (ISI). The power-allocation problem for the fading channel is identical to the power- and bit-loading problem for the ISI channel if the fading statistics is assumed to be i.i.d., and if the ISI channel is equipped with guard periods which ensure the orthogonality of subchannels.

\section{Multiple-Antenna Case}

The intuition for Knopp and Humblet's result is that a single-antenna receiver is limited by the single degree of freedom (DOF) it has. To achieve the sum capacity, only one user can transmit at a time. With multiple antennas, however, multiple dimensions may be available. So, the optimal power-control strategy may involve more than one user transmitting at the same time. Intuitively, one might suspect that the maximum number of users in this case should be bounded by the number of base-station antennas. Unfortunately, this is not the case. With multiple base-station antennas, the best 
upper bound is, in fact, related to the square of the base-station antennas. We now state the main result of this letter.

Theorem 1: In a multiple-antenna multiple-access fading channel with i.i.d. complex Gaussian channel matrices, assuming perfect channel side information at all the transmitters and at the receiver, the optimal power-control strategy that achieves the maximum sum ergodic capacity has the following property: with $m$ antennas for each user and $n$ antennas at the base-station, at any time instant, the rank of transmit signal $r_{k}$ for each users must satisfy $\sum_{k} r_{k}^{2} \leq n^{2}$. In particular, a maximum of $n^{2}$ users can transmit simultaneously.

Proof: First, consider the case in which each transmitter has one antenna and the receiver has $n$ antennas, so that the channel matrix $H_{k}(\nu)$ is an $n \times 1$ vector and the transmitter covariance is just a scalar $s_{k}(\nu)$, and the slack variable is also a scalar $\phi_{k}(\nu)$

$\lambda_{k}=H_{k}^{*}(\nu)\left(\sum_{j=1}^{K} s_{j}(\nu) H_{j}(\nu) H_{j}^{*}(\nu)+I\right)^{-1} H_{k}(\nu)+\phi_{k}(\nu)$.

The claim is that at any fading state $\nu$, only a maximum of $n^{2}$ users can have $s_{k}(\nu)>0$ and $\phi_{k}(\nu)=0$. The rest of the users must have $s_{k}(\nu)=0$ and $\phi_{k}(\nu)>0$. As in the single-antenna case, the key is to recognize that the matrix inversion in the expression is common to all users. Recall that $\lambda_{k}$ is determined by the channel fading distribution, so it can be considered fixed. We first ask whether there exists a positive definite Hermitian matrix $M$ such that $H_{k}^{*} M H_{k}=\lambda_{k}$ for more than $n^{2} H_{k}$ 's. The following lemma answers this question.

Lemma 2: Fixing positive $\lambda_{1}, \ldots, \lambda_{K}$, let $H_{1}, \ldots, H_{K}$ be $n \times 1$ random vectors whose entries are i.i.d. complex Gaussian variables. If $K>n^{2}$, then with probability one, there does not exist a positive definite Hermitian matrix $M$ such that $H_{k}^{*} M H_{k}=\lambda_{k} \forall k=1, \ldots, K$.

Proof: Let $H_{k}=\left[\begin{array}{llll}h_{k 1} & h_{k 2} & \cdots & h_{k m}\end{array}\right]^{T}$. Denote the $(i, j)$ entry of $M$ by $M_{i j}$. Since $M$ is a Hermitian matrix with complex entries (i.e., $M_{i j}=M_{j i}^{*}$ ), there are $n(n+1) / 2$ independent complex variables in the off-diagonal terms of $M$ and $n$ independent real entries on the diagonal. So, there are effectively $n^{2}$ independent real variables in $M$. To have $H_{k}^{*} M H_{k}=\lambda_{k}$, we need

$$
\sum_{i j} h_{k i} h_{k j} m_{i j}=\lambda_{k}
$$

for all $k=1, \ldots, K$. Because $H_{k}$ 's are i.i.d. Gaussian, with probability 1 , these $K$ equations are linearly independent. So a solution to (19) exists only if $K \leq n^{2}$.

Lemma 2 shows that the number of users that can transmit simultaneously is $n^{2}$ or fewer. It does not guarantee that exactly $n^{2}$ users will transmit, because the existence of a matrix $M$ satisfying (19) does not guarantee that such an $M$ can be synthesized by $s_{k}(\nu)$, as in (19).

Next, we turn our attention to the case where remote transmitters are equipped with multiple antennas. In this case, the waterfilling level is now an $m \times m$ identity matrix, and the transmitter power spectrum and the slack variables are both $m \times m$ positive semidefinite matrices. The waterfilling condition is

$\lambda_{k} I=H_{k}^{*}(\nu)\left(\sum_{j=1}^{K} H_{j}(\nu) S_{j}(\nu) H_{j}^{*}(\nu)+I\right)^{-1} H_{k}(\nu)+\Phi_{k}(\nu)$

for $k=1, \ldots, K$. Parallel to the previous development, we ask, does there exist a positive semidefinite matrix $M$ that satisfies $\lambda_{k} I=H_{k}^{*}(\nu) M H_{k}(\nu)+\Phi_{k}(\nu)$ ? The idea is again to count the number of independent equations and the number of unknowns. To satisfy the matrix equation, we need to satisfy one equation for each matrix entry. By symmetry, there are $m(m+1) / 2$ independent complex entries for each $k$. However, as the diagonal entries of the matrix equations are automatically real, the total number of independent real equations is $\mathrm{Km}^{2}$.

The number of unknown variables is counted as follows. Again, the matrix $M$ introduces $n^{2}$ DOFs. The number of unknowns introduced by the slack variable $\Phi_{k}(\nu)$ depends on its rank. $\Phi_{k}(\nu)$ are $m \times m$ matrices. An $m \times m$ complex Hermitian matrix with real diagonal entries has at most $m^{2}$ DOFs. However, if the matrix is restricted to rank $r$, the number of DOFs decreases to $m^{2}-(m-r)^{2}$. To see this, recall that a positive semidefinite Hermitian matrix can be represented by its Cholesky factorization as $L L^{*}$, where $L$ is triangular. The number of DOFs can be thought of as the number of independent variables in the triangular matrix $L$. The diagonal terms are always real. The off-diagonal terms are complex. Thus, the total number of independent entries is

$$
2\left(\sum_{i=1}^{m} i\right)-m=2 \frac{m(m+1)}{2}-m=m^{2}
$$

If an $m \times m$ matrix is of rank $r$, its Cholesky factor is an $m \times r$ triangular matrix. Again, the $(m-r)$ diagonal entries are always real, and the off-diagonal terms may be complex. So, the number of DOFs is

$$
2\left(\sum_{i=r}^{m} i\right)-(m-r)=m^{2}-(m-r)^{2}
$$

Now, the slack variables need to satisfy the complementary slackness condition

$$
\operatorname{tr}\left(\Phi_{k}(\nu) S_{k}(\nu)\right)=0
$$

So, if the transmit signal $S_{k}(\nu)$ is of rank $r_{k}$, the rank of $\Phi_{k}(\nu)$ is at most $m-r_{k}$. Therefore, each $\Phi_{k}(\nu)$ introduces at most $m^{2}-r_{k}^{2}$ extra DOFs. The total number of unknown variables is then $n^{2}$ coming from the matrix $M$, plus $m^{2}-r_{k}^{2}$ coming from each of $\Phi_{k}(\nu)$.

The matrix equations involve the channel realization $H_{k}(\nu)$, which are Gaussian random matrices. So, with probability one, these equations are independent. Thus, for a solution to exist, 
there need to be at least as many unknown variables as there are equations, so

$$
n^{2}+\sum_{k=1}^{K}\left(m^{2}-r_{k}^{2}\right) \geq K m^{2}
$$

from which the condition $\sum_{k} r_{k}^{2} \leq n^{2}$ follows.

At any time instant, a user transmits with positive power if the rank of its transmitted signal is at least 1 . Therefore, in a multiple-access scenario with $n$ receive antennas, a total of $n^{2}$ users can transmit at the same time. The power-control strategy can be thought of as choosing the "best" set of at most $n^{2}$ users, when transmitting together (using power determined by the fading state and their respective water levels), provides the highest sum capacity. This concludes the proof.

Theorem 1 establishes an upper bound on the number of simultaneous users that can transmit simultaneously in a multiple-access channel. Although the theorem does not guarantee that the bound is tight, simulation results indicate that the maximum number of simultaneous users is somewhere between $n$ and $n^{2}$. Note that this result is established assuming that $H$ has complex entries. When $H$ has real entries, Theorem 1 needs to be modified as follows. The rank bound becomes $\sum_{k} r_{k}\left(r_{k}+\right.$ $1) / 2 \leq n(n+1) / 2$. The maximum number of simultaneous users becomes $n(n+1) / 2$. Theorem 1 uses a similar proof technique as in [7]. In [7], the same technique is used to show the optimality of transmit beamforming in the multiantenna uplink. The emphasis in this letter is the structure of the optimal scheduling scheme.

\section{Optimal Downlink Power CONTROL}

The preceding result can be generalized to the downlink. The generalization relies on recent results on the sum capacity of Gaussian vector broadcast channels [8]-[11] and a key insight known as the duality of the uplink and downlink channels [11]-[13].

The duality result can be stated as follows. The sum capacity of a broadcast channel is exactly the same as the sum capacity of a dual multiple-access channel with channel matrices transposed and with a sum-power constraint imposed across all the users. Mathematically, the power-control strategy $S^{\prime}(\nu)$ that maximizes the sum ergodic capacity for a fading broadcast channel (6) can also be found by first solving for the KKT condition of a dual multiple-access channel

$$
\begin{aligned}
& H_{k}(\nu)\left(\sum_{j=1}^{K} H_{j}^{*}(\nu) D_{j}(\nu) H_{j}(\nu)+I\right)^{-1} H_{k}^{*}(\nu)+\Phi_{k}(\nu)=\lambda I \\
& \quad \int_{\nu} \sum_{j=1}^{K} \operatorname{tr}\left(D_{j}(\nu)\right) d \rho(\nu) \leq P \\
& \quad \operatorname{tr}\left(\Phi_{k}(\nu) D_{k}(\nu)\right)=0 \\
& \quad \Phi_{k}(\nu), D_{k}(\nu), \lambda_{k} \geq 0
\end{aligned}
$$

where $D_{1}(\nu), \ldots, D_{K}(\nu)$ are the transmit covariance matrices of the dual channel. Note that the only difference between the

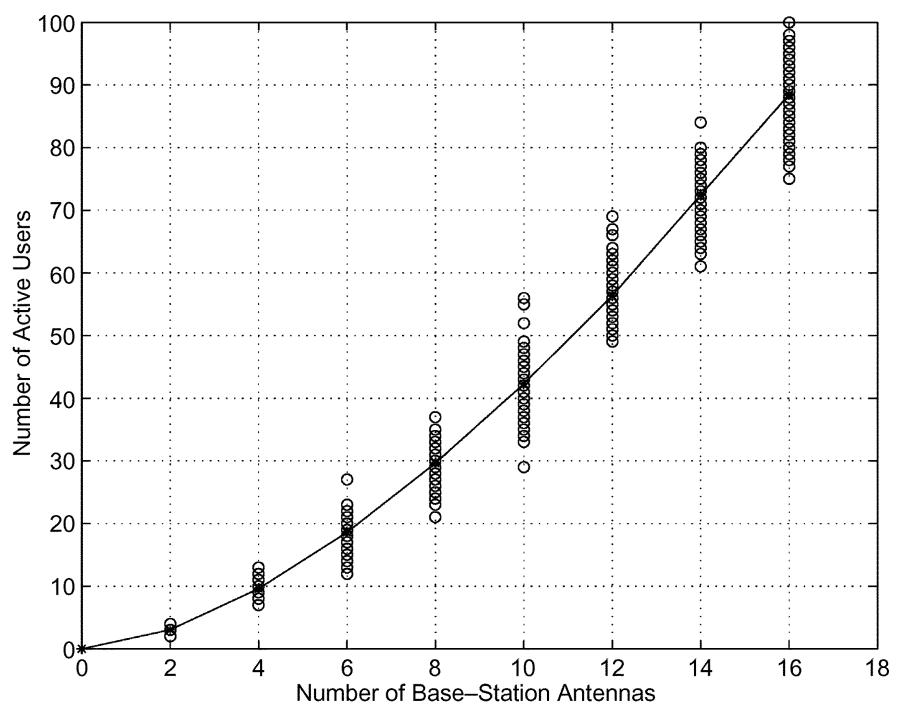

Fig. 1. Number of active users versus the number of base-station antennas over random realizations of the channel. Both the range and the average number of active users are shown.

above set of equations and (12)-(15) is that the dual channel matrices are now $H_{k}^{*}(\nu)$ and a sum-power constraint is applied across all $D_{j}(\nu)$ 's. Based on the optimal transmit covariance matrices of the dual multiple-access channel, the optimal $S^{\prime}(\nu)$ in the broadcast channel may then be found via

$$
S^{\prime}(\nu)=(\lambda I)^{-1}-\left(H^{*} \lambda D(\nu) H+\lambda I\right)^{-1} .
$$

Further, $\lambda D_{k}(\nu)$ is precisely the optimal receiver matrix at each remote user.

Corollary 1: In a multiple-antenna broadcast fading channel with i.i.d. complex Gaussian channel matrices, the optimal transmission strategy with perfect channel side information at the transmitter and at all the receivers that achieves the maximum sum ergodic capacity has the following property. With $m$ antennas for each user and $n$ antennas at the base-station, at any time instant, the rank of receiver matrix $r_{k}$ for each users must satisfy $\sum_{k} r_{k}^{2} \leq n^{2}$. In particular, a maximum of $n^{2}$ receivers can be active simultaneously.

The proof of the corollary follows the same line of argument as the proof of Theorem 1. As the waterfilling level $\lambda$ is fixed by the fading distribution, the same dimension-counting argument can be made for the dual multiple-access channel. The analysis of the number of variables versus the number of equations reveals that the rank of $D_{k}$ must satisfy $\sum_{k} r_{k}^{2} \leq n^{2}$. Now, as $\lambda D_{k}$ is the receiver matrix at the remote user, the rank bound for the dual multiple-access channel translates directly to the rank bound for the receiver matrix. In particular, at most $n^{2}$ receivers can be active at any given time.

\section{SPATIAL Diversity Versus Multiuser DiVERSITy}

In both the multiple-access fading channel and the broadcast fading channel, the number of DOFs created by multiple antennas is bounded by a quadratic function of the number of antennas. With $n$ antennas at the base-station, the maximum number of DOFs at the base-station is theoretically upper 

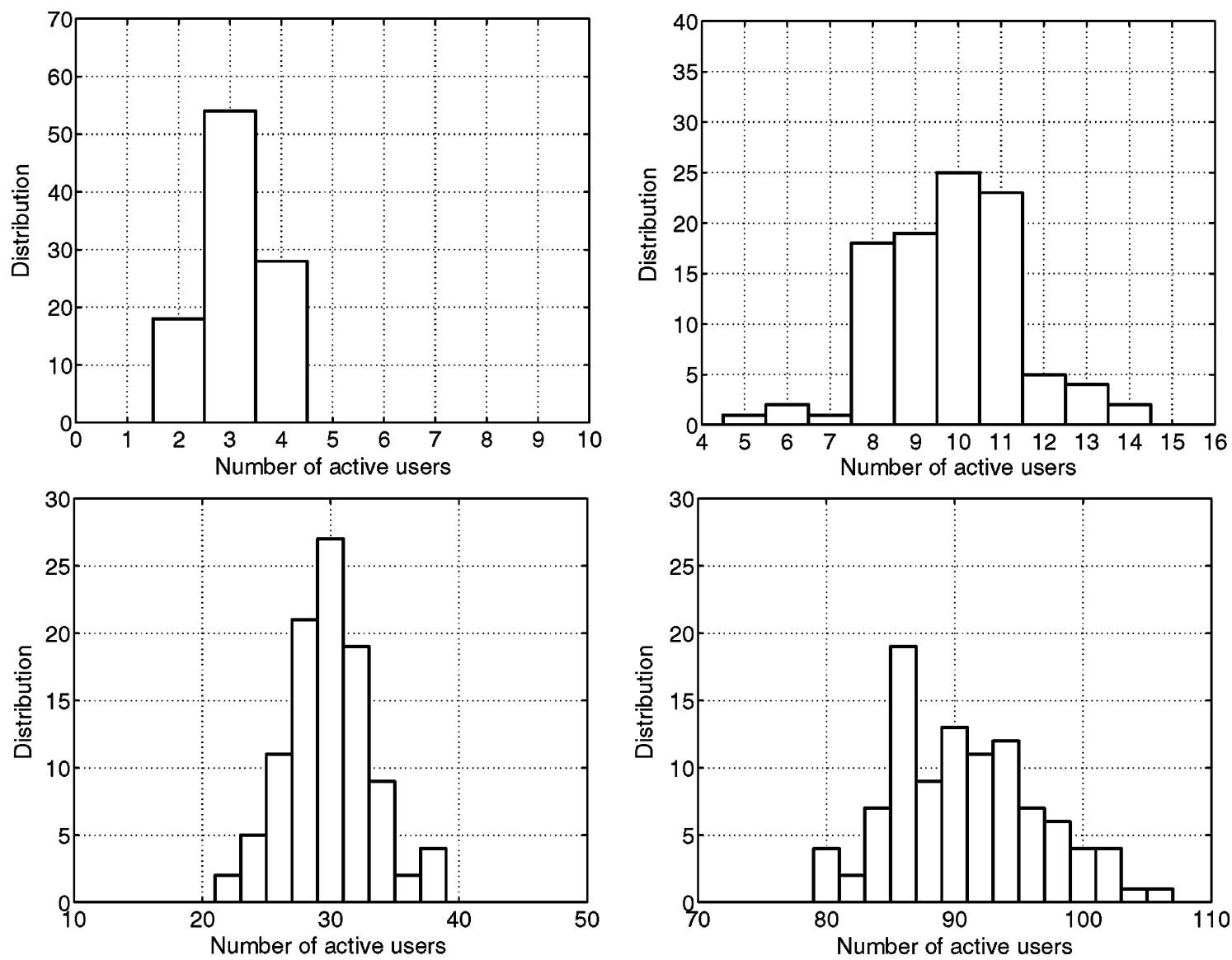

Fig. 2. Histograms of the number of active users over 100 random realizations of the channel. Top-left, top-right, bottom-left and bottom-right figures correspond to the cases of $2,4,8$, and 16 base-station antennas, respectively. The remote terminals are equipped with one antenna each.

bounded by $n^{2}$. These DOFs are to be divided among the remote users. Each remote user, with $m$ antennas, can potentially use up to $m^{2}$ DOFs. Thus, the number of base-station antennas has the effect of allowing more users to transmit simultaneously, while the number of antennas at the remote users has the opposite effect. The remote antennas have the potential to crowd out receiver dimensions, and thus prevent other users from transmitting at the same time. Such crowding out increases system sum capacity at the expense of delay and unfairness. It should be noted that such crowding out, although theoretically possible, rarely happens in an i.i.d. Gaussian channel. As was pointed out in [7], the remote users are most likely to employ a beamforming strategy which effectively reduces each remote user to a single-dimension transmission or receiving terminal.

\section{SimUlation Results}

Theorem 1 provides a theoretical upper bound on the total number of active users in a multiuser multiantenna wireless channel. Simulation results suggest that this upper bound is tight when the number of antennas at the base-station is small. Although the bound becomes loose as the number of antennas grows large, simulation results also suggest that the actual maximum number of active users appear to grow superlinearly as a function of the number of base-station antennas.

Figs. 1 and 2 show simulation results on a Rayleigh fading channel with multiple remote users, each with a single antenna and a base-station with a varying number of transmit antennas. The optimization problem for the uplink is solved using the iterative waterfilling algorithm [5]. The corresponding downlink problem is solved using a combination of iterative waterfilling and a dual-decomposition approach [14]. The number of active users versus the number of transmit antennas over 100 realizations of the channel is plotted in Fig. 1. It is seen that the theoretical bound is achieved when the number of base-station antennas is small. For example, with 2 and 4 antennas at the base-station, the number of active users is close to 4 and 16, respectively. The bound, however, does not seem to be tight when the number of base-station antennas is large.

Fig. 2 shows histograms of the number of active users over 100 random realizations of the channel for the cases of 2-16 base-station antennas. There are substantial variations among different realizations of the channel. When the number of basestation antennas is large, the number of active users is almost always several times higher than the number of antennas.

\section{CONCLUDING REMARKS}

When the base-station in a cellular system is equipped with a single antenna, the sum-rate maximizing transmission strategy consists of only a single active user. Essentially, only the user with the best channel is being served at any given time. The main point of this letter is to show that with multiple antennas at the base-station, the sum-rate maximizing strategy consists 
of multiple simultaneous active users. The maximum number of simultaneous users is typically larger than (and is bounded by the square of) the number of base-station antennas. The main result of this letter provides a dimension-counting argument for multiuser multiantenna wireless systems. There is an interesting interplay between spatial diversity and multiuser diversity. In a random propagation environment, a downlink broadcast channel with $n$ transmit antennas has up to $n^{2}$ DOFs. These dimensions are divided among the remote terminals. A remote terminal with $m$ receive antennas can use up to $m^{2}$ dimensions. The dimensions are additive, and the total number of dimensions is upper bounded by $n^{2}$. This same result applies to both uplink and downlink fading channels.

\section{REFERENCES}

[1] R. Knopp and P. A. Humblet, "Information capacity and power control in single-cell multi-user communications," in Proc. IEEE Int. Conf. Commun., 1995, pp. 331-335.

[2] E. F. Chaponniere, P. Black, J. M. Holtzman, and D. Tse, "Transmitter directed multiple receiver system using path diversity to equitably maximize throughput," U.S. Patent 6449490, Sep. 10, 2002.

[3] W. Rhee and J. M. Cioffi, "Increase in capacity of multiuser OFDM system using dynamic subchannel allocation," in Proc. IEEE Veh. Technol. Conf., Tokyo, Japan, Spring, 2000, vol. 2, pp. 1085-1089.
[4] X. Qin and R. Berry, "Exploiting multiuser diversity for medium access control in wireless networks," in Proc. 22nd Annu. Joint Conf. IEEE Comput. Commun. Soc., Mar. 2003, vol. 2, pp. 1084-1094.

[5] W. Yu, W. Rhee, S. Boyd, and J. M. Cioffi, "Iterative water-filling for Gaussian vector multiple access channels," IEEE Trans. Inf. Theory, vol. 50, no. 1, pp. 145-152, Jan. 2004.

[6] R. S. Cheng and S. Verdu, "Gaussian multiaccess channels with ISI: Capacity region and multiuser water-filling," IEEE Trans. Inf. Theory, vol. 39, no. 3, pp. 773-785, May 1993.

[7] W. Rhee, W. Yu, and J. M. Cioffi, "The optimality of beam-forming in uplink multiuser wireless systems," IEEE Trans. Wireless Commun., vol. 3, no. 1, pp. 86-96, Jan. 2004

[8] G. Caire and S. Shamai, "On the achievable throughput of a multiantenna Gaussian broadcast channel," IEEE Trans. Inf. Theory, vol. 49, no. 7, pp. 1691-1706, Jul. 2003.

[9] W. Yu and J. M. Cioffi, "Sum capacity of Gaussian vector broadcast channels," IEEE Trans. Inf. Theory, vol. 50, no. 9, pp. 1875-1892, Sep. 2004.

[10] S. Vishwanath, N. Jindal, and A. Goldsmith, "Duality, achievable rates and sum-rate capacity of Gaussian MIMO broadcast channels," IEEE Trans. Inf. Theory, vol. 49, no. 10, pp. 2658-2668, Oct. 2003.

[11] P. Viswanath and D. Tse, "Sum capacity of the vector Gaussian broadcast channel and uplink-downlink duality," IEEE Trans. Inf. Theory, vol. 49, no. 8, pp. 1912-1921, Aug. 2003.

[12] N. Jindal, S. Vishwanath, and A. Goldsmith, "On the duality of Gaussian multiple-access and broadcast channels," IEEE Trans. Inf. Theory, vol. 50, no. 5, pp. 768-783, May 2004.

[13] W. Yu, "Uplink-downlink duality via minimax duality," IEEE Trans. Inf. Theory, vol. 52, no. 2, pp. 361-374, Feb. 2006.

[14] W. Yu, "Sum capacity computation for the Gaussian vector broadcast channel," IEEE Trans. Inf. Theory, vol. 52, no. 2, pp. 754-759, Feb. 2006. 